\title{
Higher Referrals for Diabetes Education in a Medical Home Model of Care
}

\author{
William T. Manard, MD, Kevin Syberg, DrPH, Anit Behera, BS, Joanne Salas, MPH, \\ F. David Schneider, MD, Eric Armbrecht, PhD, Denise Hooks-Anderson, MD, \\ Erica Crannage, PharmD, and Jeffrey Scherrer, $P h D$
}

Background: The medical home model has been gaining attention from the health care community as a strategy for improved outcomes for management of chronic disease, including diabetes. The purpose of this study was to compare referrals for diabetes education among patients receiving care from a medical home model versus a traditional practice.

Methods: Data were obtained from a large, university-affiliated primary care patient data registry. All patients (age 18-96 years) with a diagnosis of prediabetes or diabetes and seen by a physician at least twice during 2011 to 2013 were selected for inclusion. Multivariate regression models measuring the association between medical home status and referral to diabetes education were computed before and after adjusting for covariates.

Results: A significantly $(P<.001)$ higher percentage of patients in a medical home than without a medical home $(23.9 \%$ vs $13.5 \%)$ received a referral for diabetes education. After adjusting for covariates, medical home patients were 2.7 times more likely to receive a referral for diabetes education (odds ratio, 2.70; 95\% confidence interval, 1.69-4.35).

Conclusion: Patients in a medical home model were more likely to receive referrals for diabetes education than patients in a standard university-affiliated family medicine practice. Future longitudinal designs that match characteristics of patients with a medical home with those of patients without one will provide strong evidence to determine whether referral to diabetes education is a result of the medical home model of care independent of confounding factors. ( $\mathrm{J}$ Am Board Fam Med 2016;29:377-384.)

Keywords: Diabetes Mellitus; Education, Patient; Medical Home

One of the most effective interventions for diabetes is education regarding self-management. Diabetes education, as endorsed by the American Diabetes Asso-

This article was externally peer reviewed.

Submitted 1 December 2015; revised 12 January 2016; accepted 28 January 2016.

From the Department of Family and Community Medicine, Saint Louis University School of Medicine (WTM, JSa, FDS, DH-A, JSc); the Center for Outcomes Research, Saint Louis University School of Medicine (KS, AB, EA); the Department of Pharmacy Practice, Division of Ambulatory Care, Saint Louis College of Pharmacy, Saint Louis University School of Medicine (EC).

Funding: Partial support for this work was provided by Health Resources and Services Administration grant D54HP23286, Academic and Administrative Units in Primary Care, from the U.S. Department of Health and Human Services.

Conflict of interest: none declared.

Corresponding author: Jeffrey Scherrer, $\mathrm{PhD}$, Department of Family and Community Medicine, Saint Louis University School of Medicine, 1402 South Grand, St. Louis, MO 63104 (E-mail: scherrjf@slu.edu). ciation, is important both clinically and therapeutically for patients. ${ }^{1}$ For example, diabetes education has been shown to improve blood glucose control. ${ }^{2}$ The American Diabetes Association recommended in 2015 that all patients receive diabetes self-management education and support at diagnosis and thereafter as needed. ${ }^{3}$

Several barriers to receipt of diabetes education are described in the literature. A report of Canadian primary care practices revealed that less than half of physicians made referrals, citing patient's low enthusiasm, lack of weekend and evening appointments, and inconvenience. ${ }^{4}$ The most significant reason for patients not to attend diabetes education was a belief that their diabetes knowledge was sufficient. ${ }^{5}$ In addition, some patients reported that diabetes treatment was solely the physician's responsibility. ${ }^{5}$ Cultural barriers to diabetes care could also be considered, for example, 
as reported by physicians managing Hispanic patients in southwestern New Mexico. ${ }^{6}$ Little or ineffective physician-patient communication, as well as a belief that the physician is the primary source for education, may also limit self-management education. ${ }^{7,8}$ Other general barriers include socioeconomic status (SES), cost of services, language, and transportation. ${ }^{4,7,8}$

It has been proposed that the patient-centered medical home (PCMH) model, which embraces a team-based, interdisciplinary approach to patient care, is a good model of care for diabetes because care coordination, a whole-person approach, and care management allow for greater access and follow-up, among other benefits. ${ }^{9}$ For instance, nonphysician team members can provide self-care education and care managers can track patients to ensure they follow referrals for diabetes education and other subspecialties. ${ }^{9}$ The PCMH model is an form of primary care that focuses primarily on the patient, who is the center of a medical team; decisions are made between the patient, his or her family, and health professionals to ensure the patient's best interests are met. ${ }^{10}$ The Agency for Healthcare Research and Quality divides the PCMH into 5 basic components: patient-centered, comprehensive, coordinated, accessible, and committed to quality and safety. ${ }^{10}$ The benefits of the PCMH model are that the patient's needs are satisfied, the patient is able to access care with shorter waiting times, care is organized across all elements of the broader health care system, and the patient is educated on the basics of their health. ${ }^{10}$ According to Bojadzievski and Gabbay, ${ }^{9}$ diabetes would fit well into the principles of the PCMH given its high cost, well-demonstrated quality gaps, and robust evidence-based guidelines. Therefore diabetes care can easily be aligned with the core values of the PCMH with a focus on self-management, patient empowerment, and early recognition of the importance of patient-centered care. ${ }^{9}$

The adoption of a PCMH is associated with reduced costs of diabetes care, ${ }^{11}$ a finding partly driven by fewer emergency department visits and hospitalizations. A survey of Medicaid patients with type 2 diabetes indicated that as the patient rating of a medical home's performance increased, the odds of patients in that medical home receiving guideline-concordant diabetes care increased. ${ }^{12}$ Both studies show that the benefits of a medical home may be biased by the providers who seek to make their practice a medical home. As eluded to by Wang et al, ${ }^{11}$ these providers may be personally incentivized to aggressively pursue quality indicators more than nonmedical home providers. ${ }^{11,12}$

Because of the sparse literature on PCMHs and diabetes, and because of limitations related to provider self-selection to make their practice a medical home and subsequently adopt aggressive pursuit of quality indicators, we sought to expand the nascent research by using a retrospective cohort designone not biased by provider self-selection-to determine whether referral to diabetes education documented in the medical record was significantly more common among family medicine (FM) patients with prediabetes or diabetes who are enrolled in a PCMH compared with non-PCMH patients.

\section{Methods \\ Participants}

Clinical data for 27,225 patients were obtained from the Saint Louis University Department of Family and Community Medicine's Primary Care Patient Data (PCPD) Registry. This registry was developed by a programmer who extracted electronic medical record (EMR) files, de-identified the records, and sent the files to the team biostatistician. Patient data files captured all visits to FM and general internal medicine (GIM) clinics at a large academic medical practice located in the St. Louis, Missouri, metropolitan area. The PCPD Registry contains 10,994 unique FM patients and 16,231 unique GIM patients who had at least 1 visit between July 1, 2008, and July 31, 2013. Because the PCMH program was implemented in the FM practice in 2011, the sample for this study is patient visits from 2011 to 2013.

Study variables were created from International Classification of Diseases, 9th Revision, Clinical Modification (ICD-9-CM) codes, laboratory results, prescription orders, Current Procedure Terminology codes (a standard numeric coding scheme used in the United States to describe medical procedures), social history, vital signs, and EMR-specific indicators of referral to diabetes education. The PCPD Registry has been used in several clinical epidemiology and health services research studies, where additional details regarding the PCPD Registry are available. ${ }^{13-17}$ The institutional review board approved the creation of this retrospective cohort and its use for primary care research. 
We included in this study only patient encounters in FM because GIM does not have the PCMH program, and the demographic and clinical characteristics of GIM substantially differ from FM. ${ }^{13-17}$

\section{Diabetes and Prediabetes Definitions}

For this study, patients with prediabetes were defined by ICD-9-CM code 790.29 or an $\mathrm{HbA}_{1 \mathrm{c}}$ value of $5.7 \%$ to $6.4 \%(39-46 \mathrm{mmol} / \mathrm{mol})$; patients with type 2 diabetes were defined by the presence of an ICD-9-CM code (250.x0, 250.x2). Using these definitions, the study population included 962 patients aged 18 to $>89$ years, of whom $44.1 \%$ $(\mathrm{n}=424)$ had prediabetes and $55.9 \%(\mathrm{n}=538)$ had diabetes.

\section{Medical Home}

Beginning in January 2011, the academic FM medical group opened a PCMH-model program in partnership with the university administration, which made the program available, on a voluntary basis, to all employees, spouses, and dependents. The medical home program receives a stipend from Human Resources to provide care management services. The stipend agreement required that beneficiaries receive access to a PCMH-model practice. Beyond the fixed stipend, there are incentives for meeting certain chronic disease measures (blood sugar control, depression screening, etc.) that are responsive to care management services. By meeting these measures, the program can achieve additional funding within the terms of the agreement.

The university employs approximately 4500 people and provides an employer-sponsored health insurance plan for about 7500 people. The PCMH program operated at 3 sites in the metropolitan area. By December 2013, 155 patients with prediabetes or diabetes were members of the medical home, and 807 patients with prediabetes or diabetes received non-PCMH care from the universityaffiliated family medicine practice.

\section{Diabetes Education}

Patients were coded as having been referred to diabetes education if they had in the EMR the institution-specific referral codes indicating either referral to a dietitian/nutritionist and/or referral to diabetic education. Indications for referral to diabetes education were generally a new diagnosis of diabetes, adherence challenges, and difficulty in achieving glycemic control.

\section{Covariates}

Covariates hypothesized to be associated with referral for diabetes education and correlated with medical home enrollment included demographic variables (age, sex, and race); diabetes management (A1c control, A1c tested, cholesterol tested, microalbumin tested); diabetes-related comorbidity (hyperlipidemia, hypertension, vascular disease); and risk factors for poor diabetes outcomes (obesity and smoking status). Detection bias (the volume of health care utilization) was also hypothesized to be associated with diabetes education; the more encounters with the health care system a patient had, the more likely a referral could be made.

The variable of race was re-coded as white or nonwhite. SES was determined based on the zip code of residence using an index that uses information from 7 measures of SES obtained from 5-year (2009-2013) census estimates from the American Community Survey. ${ }^{18}$ These 7 variables include the percentage of households with income below the poverty level, receiving public assistance, and with an annual income $<\$ 35,000$; the percentage of adult men aged 20 to 64 years not in the labor force and those aged $\geq 25$ years with less than a high school education; and the logs of the median household income and of the median value of single-family homes. ${ }^{18}$ Based on the SES factor score distribution in the PCPD Registry, eligible patients were assigned into quartiles (eg, lowest, low-middle, upper-middle, and highest SES).

We classified A1c as controlled (A1c $<7 \%[<53$ $\mathrm{mmol} / \mathrm{mol}]$ ), uncontrolled (A1c $\geq 7 \%[\geq 53 \mathrm{mmol} /$ mol]), or unknown. Health care utilization was measured by the average number of clinic visits per month for each patient; it was modeled as a binary variable measuring high utilizers (top 25\% of primary care office visit frequency among all included patients) versus non-high utilizers (bottom $75 \%$ of primary care office visit frequency among all included patients). ICD-9-CM codes were used to define the presence of hyperlipidemia (272.0-272.4), hypertension (401.x), and vascular disease (402-405, 410-417, 420-429, 430-438). Obesity was defined by body mass index $\geq 30.0$ $\mathrm{kg} / \mathrm{m}^{2}$ and/or an ICD-9-CM diagnosis (codes 278.00 and/or 278.01). Smoking status was obtained from the patient's social history or an ICD- 
9-CM diagnosis for nicotine dependence (V15.82, 305.1) and categorized as never, past, or current. diabetes referral were included in a multivariable model used to calculate adjusted odds ratios.

\section{Analysis}

All analyses were conducted using SAS version 9.4 (SAS, Inc., Cary, NC). The prevalence of covariates was calculated and compared between those referred and not referred to diabetes education and between medical home and non-medical home patients using $\chi^{2}$ or independent-samples $t$ test. A crude bivariate odds ratio and $95 \%$ confidence interval for the association of medical home and referral to diabetes education was calculated using binary logistic regression. Variables with bivariate associations $(P<.05)$ with both medical home and

\section{Results}

Characteristics of non-medical home and medical home patients are shown in Table 1. Non-medical home patients were significantly older, on average, than medical home patients $(P<.0001)$. White race was significantly more common among medical home patients than in non-medical home patients $(P<.0001)$. About two-thirds of both medical home and non-medical home patients were women. Medical home patients were less often in the highest and lowest SES quartiles compared

Table 1. Attributes of Patients Receiving Primary Health Care Services from a University-Affiliated Medical Home Model versus a Standard Family Medicine Practice $(n=962)$

\begin{tabular}{|c|c|c|c|c|}
\hline & Overall $(\mathrm{N}=962)$ & No medical home $(\mathrm{n}=807)$ & Medical home $(\mathrm{n}=155)$ & $P$ Value \\
\hline Mean age (years), SD & $55.9(13.5)$ & $56.7(13.8)$ & $52.1(10.9)$ & $<.0001$ \\
\hline Nonwhite race & $44.3(426)$ & $47.1(380)$ & $29.7(46)$ & $<.0001$ \\
\hline Female sex & $68.0(654)$ & $68.6(554)$ & $64.5(100)$ & .312 \\
\hline \multicolumn{5}{|l|}{ SES index (based on zip code) } \\
\hline Lowest & $232(24.1)$ & $204(25.3)$ & $28(18.1)$ & \\
\hline Lower middle & $151(15.7)$ & $127(15.7)$ & $24(15.5)$ & .011 \\
\hline Upper middle & $260(27.0)$ & $202(25.0)$ & $58(37.4)$ & \\
\hline Highest & $319(33.2)$ & $274(34.0)$ & $45(29.0)$ & \\
\hline \multicolumn{5}{|l|}{ Diabetes diagnosis } \\
\hline Prediabetes & $44.1(424)$ & $42.9(346)$ & $50.3(78)$ & .087 \\
\hline Diabetes & $55.9(538)$ & $57.1(461)$ & $49.7(77)$ & \\
\hline Referral to dietitian/education & $15.2(146)$ & $13.5(109)$ & $23.9(37)$ & .001 \\
\hline \multicolumn{5}{|l|}{$\mathrm{HbA}_{1 \mathrm{c}}$ control } \\
\hline Controlled & $51.8(498)$ & $50.2(405)$ & $60.0(93)$ & .081 \\
\hline Uncontrolled & $29.5(284)$ & $30.5(246)$ & $24.5(38)$ & \\
\hline Unknown & $18.7(180)$ & $19.3(156)$ & $15.5(24)$ & \\
\hline $\mathrm{HbA}_{1 \mathrm{c}}$ tested & $88.5(851)$ & $87.7(708)$ & $92.3(143)$ & .106 \\
\hline Cholesterol tested & $82.7(796)$ & $82.2(663)$ & $85.8(133)$ & .271 \\
\hline Microalbumin tested & $45.8(441)$ & $45.5(367)$ & $47.7(74)$ & .604 \\
\hline $\begin{array}{l}\text { Patients with high service utilization } \\
\text { (top } 25 \% \text { ) }\end{array}$ & $50.8(489)$ & $48.7(393)$ & $61.9(96)$ & .003 \\
\hline \multicolumn{5}{|l|}{ Chronic diseases } \\
\hline Vascular disease & $23.8(229)$ & $25.4(205)$ & $15.5(24)$ & .008 \\
\hline Hyperlipidemia & $51.0(491)$ & $50.9(411)$ & $51.6(80)$ & .876 \\
\hline Hypertension & $68.4(658)$ & $69.1(558)$ & $64.5(100)$ & .256 \\
\hline Obesity & $75.2(723)$ & $73.6(594)$ & $83.2(129)$ & .011 \\
\hline \multicolumn{5}{|l|}{ Smoking status } \\
\hline Never & $56.8(546)$ & $56.6(457)$ & $57.4(89)$ & .968 \\
\hline Past (quit) & $25.8(248)$ & $25.8(208)$ & $25.8(40)$ & \\
\hline Current & $17.4(168)$ & $17.6(142)$ & $16.8(26)$ & \\
\hline
\end{tabular}

Data are \% (n) unless otherwise indicated.

$\mathrm{SD}$, standard deviation; SES, socioeconomic status. 
with non-medical home patients and were more often in the upper-middle SES quartile $(P=.011)$.

Among medical home patients $(\mathrm{n}=155), 50.3 \%$ had prediabetes and $49.7 \%$ had type 2 diabetes. Non-medical home patients $(\mathrm{n}=807)$ had a lower prevalence of prediabetes $(42.9 \%)$ and a higher prevalence of type 2 diabetes $(57.1 \%)$. A significantly higher prevalence of medical home patients were referred to a dietitian/diabetes educator compared with non-medical home patients $(23.9 \%$ vs $13.5 \% ; P<.0001)$.

Medical home patients were more likely to be high health care utilizers $(P=.003)$, less likely to have vascular disease $(P=.08)$, and more likely to be obese $(P=.011)$ compared with non-medical home patients. The prevalence of diabetes quality indicators $\left(\mathrm{HbA}_{1 \mathrm{c}}\right.$ tested, cholesterol tested, and microalbumin tested) did not significantly differ between medical home and non-medical home patients. The prevalence of diagnoses of hyperlipidemia and hypertension and smoking status did not significantly differ between medical home and nonmedical home patients.

Patient characteristics by referral to diabetes education are shown in Table 2. Patients with diabetes were referred more often than patients with prediabetes $(P<.0001)$. Nonwhite race and high clinic utilization were significantly more common among those referred compared with those not referred $(P<.0001)$. Patients referred to education were evenly distributed across SES quartiles but, compared with nonreferred patients, those receiving a referral were less often from the highest SES group $(P<.001)$.

Referred patients were significantly more likely to have a diagnosis of hypertension $(P=.019)$ and to be obese $(P<.0001)$. Patients referred to diabetes education were significantly more likely to have had $\operatorname{HbA}_{1 \mathrm{c}}(P=.002)$, cholesterol $(P=.29)$, and microalbumin $(P<.0001)$ tested. Referred patients were also significantly more likely to have uncontrolled $\mathrm{HbA}_{1 \mathrm{c}}(P<.0001)$.

The results of crude and adjusted logistic regression models are shown in Table 3. The unadjusted bivariate association of medical home and diabetes referral shows that medical home patients had twice the odds of referral to diabetes education than non-medical home patients (odds ratio [OR], 2.01; 95\% confidence interval, 1.32-3.06). After adjusting for diabetes versus prediabetes, race, SES, volume of health care utilization, vascular disease,
Table 2. Characteristics of Family Medicine Patients, * by Referral to Diabetes Education $(n=962)$

\begin{tabular}{|c|c|c|c|}
\hline Characteristic & $\begin{array}{l}\text { No Referral } \\
(\mathrm{n}=816)\end{array}$ & $\begin{array}{l}\text { Referral } \\
(\mathrm{n}=146)\end{array}$ & $P$ Value $^{\dagger}$ \\
\hline \multicolumn{4}{|l|}{ Diabetes diagnosis } \\
\hline Prediabetes & $48.5(396)$ & $19.2(28)$ & \multirow[t]{2}{*}{$<.0001$} \\
\hline Diabetes & $51.5(420)$ & $80.8(118)$ & \\
\hline $\begin{array}{l}\text { Age (years), mean } \\
\text { (SD) }\end{array}$ & $56.2(13.8)$ & $54.7(11.9)$ & .215 \\
\hline Nonwhite race & $41.2(336)$ & $61.6(90)$ & $<.0001$ \\
\hline Female sex & $67.4(550)$ & $71.2(104)$ & .361 \\
\hline \multicolumn{4}{|l|}{$\begin{array}{l}\text { SES index (based on } \\
\text { zip code) }\end{array}$} \\
\hline Lowest & $192(23.5)$ & $40(27.4)$ & \multirow[t]{4}{*}{.001} \\
\hline Lower middle & $115(14.1)$ & $36(24.7)$ & \\
\hline Upper middle & $221(27.1)$ & $39(26.7)$ & \\
\hline Highest & $288(35.3)$ & $31(21.2)$ & \\
\hline Utilization (top 25\%) & $47.9(391)$ & $67.1(98)$ & $<.0001$ \\
\hline \multicolumn{4}{|l|}{ Chronic disease } \\
\hline Hyperlipidemia & $51.6(421)$ & $47.9(70)$ & .417 \\
\hline Hypertension & $66.9(546)$ & $76.7(112)$ & .019 \\
\hline Vascular Disease & $24.0(196)$ & $22.6(33)$ & .711 \\
\hline Obesity & $72.3(590)$ & $91.1(133)$ & $<.0001$ \\
\hline \multicolumn{4}{|l|}{ Testing } \\
\hline $\mathrm{HbA}_{1 \mathrm{c}}$ & $87.1(711)$ & $95.9(140)$ & .002 \\
\hline Cholesterol & $81.6(666)$ & $89.0(130)$ & .029 \\
\hline Microalbumin & $41.3(337)$ & $71.2(104)$ & $<.0001$ \\
\hline \multicolumn{4}{|l|}{$\mathrm{HbA}_{1 \mathrm{c}}$ Control } \\
\hline Controlled & $55.4(452)$ & $31.5(46)$ & \multirow{3}{*}{$<.0001$} \\
\hline Uncontrolled & $25.0(204)$ & $54.8(80)$ & \\
\hline Unknown & $19.6(160)$ & $13.7(20)$ & \\
\hline \multicolumn{4}{|l|}{ Smoking status } \\
\hline Never & $57.1(466)$ & $54.8(547)$ & \multirow{3}{*}{.059} \\
\hline Past (quit) & $26.6(217)$ & $21.2(31)$ & \\
\hline Current & $16.3(133)$ & $24.0(35)$ & \\
\hline
\end{tabular}

Data are \% (n) unless otherwise indicated.

*Patients had at least 2 visits in 2011-2013, were aged 18 to $>89$ years, and had prediabetes or diabetes.

${ }^{+} P<.05$ is significant.

$\mathrm{SD}$, standard deviation; SES, socioeconomic status.

and $\mathrm{HbA}_{1 \mathrm{c}}$ control, being in the medical home was significantly associated with a greater odds of referral to diabetes education (OR, 2.70; 95\% confidence interval, 1.68-4.33). All covariates except vascular disease were positively associated with referral to diabetes education.

\section{Discussion}

We observed a significant association between enrollment in the medical home and referral to diabetes education among a cohort of 962 patients with prediabetes and diabetes. Even after control- 
Table 3. Logistic Regression Models of the Association of Medical Home Status and Referral to Diabetes Education among Family Medicine Patients* $(n=962)$

\begin{tabular}{lcc}
\hline Variable & Model 1 (Crude) & Model 2 (Adjusted) \\
\hline Medical home & 2.01 (1.32-3.06) & $2.70(1.68-4.33)$ \\
Diabetes diagnosis & & 1.00 \\
$\quad$ Prediabetes & $2.17(1.27-3.70)$ \\
$\quad$ Diabetes & $2.20(1.41-3.42)$ \\
Nonwhite race & \\
SES index (based on & \\
$\quad$ zip code) & 1.00 \\
Lowest & $1.69(0.98-2.93)$ \\
$\quad$ Lower middle & $1.07(0.62-1.84)$ \\
$\quad$ Upper middle & $0.94(0.52-1.69)$ \\
Highest & $1.87(1.25-2.80)$ \\
High clinic utilization & $0.79(0.50-1.26)$ \\
Vascular disease & \\
HbA ${ }_{1 \mathrm{c}}$ control & 1.00 \\
Controlled & $2.51(1.54-4.09)$ \\
Uncontrolled & $1.29(0.72-2.31)$ \\
Unknown & \\
\hline
\end{tabular}

Data are odds ratio (95\% confidence interval).

*Patients had at least 2 visits in 2011-2013, were aged 18 to $>80$ years, and had prediabetes or diabetes.

SES, socioeconomic status.

ling for $\mathrm{HbA}_{1 \mathrm{c}}$ levels, SES, and health care utilization, medical home patients were 2.7 times more likely to be referred than non-medical home patients. We are not aware of previous studies of medical home participation and referral to diabetes education in practices caring for both medical home and non-medical home patients. However, our results are consistent with prior studies indicating that patients who perceive care consistent with a high-performing medical home were significantly more likely to be referred to diabetes education. ${ }^{12}$ The association between medical home and diabetes education is also consistent with evidence that the cost of diabetes care is lower for medical home patients. ${ }^{11}$ The largest source of cost savings (44\%) associated with managing diabetes in a medical home is the reduction in hospitalizations. ${ }^{11}$ We speculate that diabetes education contributes to cost savings because education programs for diabetes have been reported to result in fewer emergency department visits and hospitalizations. ${ }^{19}$

The current evidence suggests that the medical home itself, independent of patient illness, accounts for referral to education. First, the OR obtained for the effect of medical home status remained significant after controlling for covariates, several of which were more prevalent among non-medical home patients and were positively associated with diabetes education. Specifically, diabetes compared with prediabetes, nonwhite race, and uncontrolled $\mathrm{HbA}_{1 \mathrm{c}}$ were all more prevalent among non-medical home patients and positively associated with referral to diabetes education.

These results are similar to those of our previous study in which we determined that African American patients were more likely than whites to receive referrals to diabetes education in a larger GIM + FM patient sample. ${ }^{13}$ As in the current study, we found that African American race, uncontrolled $\mathrm{HbA}_{1 \mathrm{c}}$, diabetes versus prediabetes, and higher health care utilization were associated with greater odds of referral to diabetes education. While the current study indicates better performance by the PCMH, the percentage of PCMH patients with diabetes or prediabetes who received a referral reached was merely $23.9 \%$. Clinical staff acknowledge that there is substantial room for continued improvement. The results indicating a greater odds of referral for patients with diabetes compared with prediabetes and for those with uncontrolled versus controlled $\mathrm{HbA}_{1 \mathrm{c}}$, suggest that health care providers may be waiting until $\mathrm{HbA}_{1 \mathrm{c}}$ levels are elevated to make referrals to diabetes education. The promotion of early education for patients who have prediabetes or lower $\mathrm{HbA}_{1 \mathrm{c}}$ levels may be an important indicator of a preventive approach by a PCMH.

There are several potential causes for the higher prevalence of referrals to diabetes education among the medical home patient population. First, 1 of the central tenets of the PCMH model is coordination of care ${ }^{20}$; open communication and close proximity between the diabetes educator and the primary care provider makes ongoing referrals more likely. Second, in model described here, patients are identified as members of the medical home program using a marker within the EMR; this visual cue may increase the likelihood of referral simply by reminding the provider that the patient is a member of the program. ${ }^{21}$ Finally, medical home described here provided contractual encouragement to improve chronic disease management; because of this, staff may have been more likely to encourage providers to refer such patients. This incentive complicates the interpretation of our results because we 
are unable to determine which component of the medical home, including incentives from Human Resources, influenced provider decisions to refer patients to diabetes education.

\section{Limitations and Conclusions}

Medical homes in FM may result in improved patient education about diabetes self-management and subsequently improve diabetes outcomes. The results presented here should be interpreted in the context of several limitations. Our data contain only referrals, and we do not know what percentage of patients actually made a visit to a diabetes educator, nor the number of visits. It is also possible that providers discussed diabetes education without recording a referral in the EMR. Such misclassification should have minimal impact on our findings because it would be rare and random among both medical home and non-medical home patients. $\mathrm{Pa}$ tient data are limited to a Midwestern metropolitan area and to academic medical practice clinics, and it is not known whether results from other parts of the United States and nonacademic settings would be similar. Future studies are needed comparing large numbers of medical homes implemented with and adherence to the same structure to confirm our findings and to determine whether our results apply to both academic and nonacademic medicine. The eligible patient population for medical home enrollment was limited to the university's employees and their dependents, which limits generalizability, most notably to populations with employer-sponsored health insurance and known coverage for diabetes education. The crosssectional nature of our study does not allow conclusions about causality.

\section{Conclusion}

The choice of provider and to enroll in a medical home is not random. Factors that contribute to choosing a medical home for primary care, such as a personal orientation toward health, might contribute to seeking referral to diabetes education. Future longitudinal designs that match characteristics medical home to non-medical home patient will provide strong evidence to determine whether referral to diabetes education is due to the medical home model of care, independent of confounding factors.

\section{References}

1. Funnell MM, Brown TL, Childs BP, et al. National standards for diabetes self-management education. Diabetes Care 2012;35(Suppl 1):S101-8.

2. Sperl-Hillen J, Beaton S, Fernandes O, et al. Comparative Effectiveness of patient education methods for type 2 diabetes. Arch Intern Med 2011;171:200110.

3. Powers MA, Bardsley J, Cypress M, et al. Diabetes self-management education and support in type 2 diabetes: a joint position statement of the American Diabetes Association, the American Association of Diabetes Educators, and the Academy of Nutrition and Dietetics. Diabetes Care 2015;38:1372-82.

4. Gucciardi E, Chan VW-S, Fortugno M, Khan S, Horodezny S, Swartzack SJ. Primary care physician referral patterns to diabetes education programs in Southern Ontario, Canada. Can J Diabetes 2011;35: 262-8.

5. Schäfer I, Küver C, Wiese B, Pawels M, van den Bussche H, Kaduszkiewicz H. Identifying groups of nonparticipants in type 2 diabetes mellitus education. Am J Manag Care 2013;19:499-506.

6. McCloskey J, Flenniken D. Overcoming cultural barriers to diabetes control: a qualitative study of Southwestern New Mexico Hispanics. J Cult Divers 2010;17:110-5.

7. Peyrot M, Rubin RR. Access to diabetes self-management education. Diabetes Educ 2008;34:90-7.

8. Rätsep A, Oja I, Kalda R, Lember M. Family doctors' assessment of patient- and health care systemrelated factors contributing to non-adherence to diabetes mellitus guidelines. Prim Care Diabetes 2007; $1: 93-7$.

9. Bojadzievski T, Gabbay RA. Patient-centered medical homes and diabetes. Diabetes Care 2011;34: 1047-53.

10. Patient-Centered Primary Care Collaborative. Defining the medical home. Available from: https:// www.pcpcc.org/about/medical-home. Accessed September, 2014.

11. Wang QC, Chawla R, Colombo CM, Snyder RL, Nigam S. Patient-centered medical home impact on health plan members with diabetes. J Public Health Manag Pract 2014;20:E12-20.

12. Stevens GD, Shi L, Vane C, Peters AL. Do experiences consistent with a medical-home model improve diabetes care measures reported by adult Medicaid patients? Diabetes Care 2014;37:2565-71.

13. Hooks-Anderson DA, Crannage EF, Salas J, Scherrer JF. Race and referral to diabetes education in primary care patients with prediabetes and diabetes. Diabetes Educ 2015;41:281-9.

14. Brieler J, Scherrer JF, Salas J. Differences in prescribing patterns for anxiety and depression between general internal medicine and family medicine. J Affect Disord 2014;172C:153-8. 
15. Scherrer JF, Salas J, Brieler J, Miller BJ, Meyer D, Schneider FD. Depression leads to incident vascular disease: evidence for the relevance to primary care. Fam Pract 2015;32:147-51.

16. Schoen MW, Salas J, Scherrer JF, Buckhold F. Cholesterol treatment and changes in guidelines in an academic medical practice. Am J Med 2015;128: 403-9.

17. Devkota B, Salas J, Sayavong S, Scherrer JF. Use of an online patient portal and glucose control in primary care patients with diabetes. Popul Health Manag 2015 Aug 3 [E-pub ahead of print].

18. Roblin DW. Validation of a neighborhood SES index in a managed care organization. Med Care 2013;51:e1-8.

19. Wong CK, Wong WC, Wan YF, Chan AK, Chan
FW, Lam CL. Effect of a structured diabetes education programme in primary care on hospitalizations and emergency department visits among people with type 2 diabetes mellitus: results from the Patient Empowerment Programme. Diabet Med 2015 October 3 [E-pub ahead of print]. doi: 10.1111/dme.12969.

20. Agency for Healthcare Research and Quality. Defining the PCMH. Available from: https://pcmh. ahrq.gov/page/defining-pcmh/. Accessed March 26, 2016.

21. HealthIT.gov. Strategies for optimizing an EHR system. Available from: https://www.healthit.gov/ providers-professionals/implementation-resources/ strategies-optimizing-ehr-system/. Accessed March 26, 2016. 